\title{
76.
}

\section{ON THE TRIPLE TANGENT PLANES OF SURFACES OF THE THIRD ORDER.}

[From the Cambridge and Dublin Mathematical Journal, vol. IV. (1849), pp. 118-132.]

A SURFACE of the third order contains in general a certain number of straight lines. Any plane through one of these lines intersects the surface in the line and in a conic, that is in a curve or system of the third order having two double points. Such a plane is therefore a double tangent plane of the surface, the double points (or points where the line and conic intersect) being the points of contact. By properly determining the plane, the conic will reduce itself to a pair of straight lines. Here the plane intersects the surface in three straight lines, that is in a curve or system of the third order having three double points, and the plane is therefore a triple tangent plane, the three double points or points of intersection of the lines taken two and two together being the points of contact. The number of lines and triple tangent planes is determined by means of a theorem very easily demonstrated, viz. that through each line there may be drawn five (and only five) triple tangent planes. Thus, considering any triple tangent plane, through each of the three lines in this plane there may be drawn (in addition to the plane in question) four triple tangent planes: these twelve new planes give rise to twenty-four new lines upon the surface, making up with the former three lines, twenty-seven lines upon the surface. It is clear that there can be no lines upon the surface besides these twenty-seven; for since the three lines upon the triple tangent plane are the complete intersection of this plane with the surface, every other line upon the surface must meet the triple tangent plane in a point upon one of the three lines, and must therefore lie in a plane passing through one of these lines, such plane (since it meets the surface in two lines and therefore in a third line) being obviously a triple tangent plane. Hence the whole number of lines upon the surface is twenty-seven; and it immediately follows that the number of triple tangent planes is forty-five. The number of lines upon the surface may also be obtained by the following method, which has the advantage of not assuming 
a priori the existence of a line upon the surface. Imagine the cone having for its vertex a given point not upon the surface and circumscribed about the surface, every double tangent plane of the cone is also a double tangent plane of the surface, and therefore intersects the surface in a straight line (and a conic). And, conversely, if there be any line upon the surface, the plane through this line and the vertex of the cone will be a double tangent plane of the cone. Hence the number of double tangent planes of the cones is precisely that of the lines upon the surface. By the theorems in $\mathrm{Mr}$ [Dr] Salmon's paper "On the degree of a surface reciprocal to a given one," Journal, vol. II. [1847] p. 65, the cone is of the sixth order and has no double lines and six cuspidal lines: hence by the formula in Plücker's "Theorie der algebraischen Curven," [1839] p. 211, stated so as to apply to cones instead of plane curves, viz. $n$ being the order, $x$ the number of double lines, $y$ that of the cuspidal lines, $u$ that of the double tangent planes, then

$$
u=\frac{1}{2} n(n-2)\left(n^{2}-9\right)-(2 x+3 y)\left(n^{2}-n-6\right)+2 x(x-1)+6 x y+\frac{9}{2} y(y-1),
$$

the number of double tangent planes is twenty-seven, which is therefore also the number of lines upon the surface.

Suppose the equation of one of the triple tangent planes to be $w=0$, and let $x=0, y=0$, be the equation of any two triple tangent planes intersecting the plane $w=0$ in two of the lines in which it meets the surface. Let $z=0$ be the equation of a triple tangent plane meeting $w=0$ in the remaining line in which it intersects the surface. The equation of the surface of the third order is in every case of the form $w P+k x y z=0, P$ being a function of the second order, but of the four different planes which the equation $z=0$ may be supposed to represent, one of them such that the function $P$ resolves itself into the product of a pair of factors, and for the remaining three this resolution into factors does not take place. This will be obvious from the sequel: at present I shall suppose that the plane $z=0$ is of the latter class, or that $P=0$ represents a proper surface of the second order. Since $x=0, y=0, z=0$, are treble tangent planes of the surface, each of these planes must be a tangent plane of the surface of the second order $P=0$, and this will be the case if we assume

$P=x^{2}+y^{2}+z^{2}+w^{2}$

$$
+y z\left(m n+\frac{1}{m n}\right)+z x\left(n l+\frac{1}{n l}\right)+x y\left(l m+\frac{1}{l m}\right)+x w\left(l+\frac{1}{l}\right)+y w\left(m+\frac{1}{m}\right)+z w\left(n+\frac{1}{n}\right) ;
$$

and considering $x, y, z$ and $w$ as each of them implicitly containing an arbitrary constant, this is the most general function which satisfies the conditions in question.

We are thus led to the equation of the surface of the third order:

$$
\begin{aligned}
& U=w\left\{x^{2}+y^{2}+z^{2}+w^{2}+\right. \\
& \left.y z\left(m n+\frac{1}{m n}\right)+z x\left(n l+\frac{1}{n l}\right)+x y\left(l m+\frac{1}{l m}\right)+x w\left(l+\frac{1}{l}\right)+y w\left(m+\frac{1}{m}\right)+z w\left(n+\frac{1}{n}\right)\right\}+k x y z=0 .
\end{aligned}
$$


I have found that by expressing the parameter $k$ in the particular form

$$
k=\frac{p^{2}-\left(l m n-\frac{1}{l m n}\right)^{2}}{2\left(p-l m n-\frac{1}{l m n}\right)},
$$

or, as this equation may be more conveniently written,

$$
k=\frac{p^{2}-\beta^{2}}{2(p-\alpha)} ; \quad \alpha=\operatorname{lm} n+\frac{1}{l m n}, \quad \beta=\operatorname{lm} n-\frac{1}{l m n},\left({ }^{1}\right)
$$

the equations of all the planes are expressible in a rational form. These equations are in fact the following: [ $\mathrm{I}$ have added, here and in the table p. 450, the reference numbers $12^{\prime}, 23^{\prime}$, \&c. constituting a different notation for the lines and planes.]

$(w) \quad w=0$.

( $\theta) \quad l x+m y+n z+w\left[1+\frac{1}{k}\left(l-\frac{1}{l}\right)\left(m-\frac{1}{m}\right)\left(n-\frac{1}{n}\right)\right]=0$,

(ब) $\frac{x}{l}+\frac{y}{m}+\frac{z}{n}+w\left[1+\frac{1}{k}\left(l-\frac{1}{l}\right)\left(m-\frac{1}{m}\right)\left(n-\frac{1}{n}\right)\right]=0$,

(x) $\quad x=0$,

(y) $\quad y=0$,

(z) $z=0$,

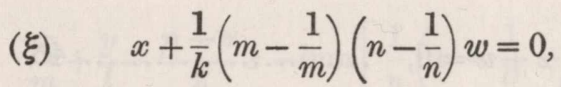

( $) \quad y+\frac{1}{k}\left(n-\frac{1}{n}\right)\left(l-\frac{1}{l}\right) w=0$,

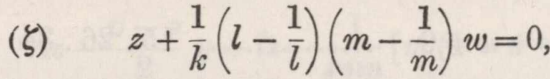

(f) $l x+\frac{y}{m}+\frac{z}{n}+w=0$,

(g) $\frac{x}{l}+m y+\frac{z}{n}+w=0$,

(h) $\frac{x}{l}+\frac{y}{m}+n z+w=0$,

${ }^{1}$ A somewhat more elegant form is obtained by writing $p=2 q+a$; this gives

$$
k=\frac{2}{q} \cdot(q+l m n)\left(q+\frac{1}{l m n}\right), \& c .
$$


(द) $\quad \frac{x}{l}+m y+n z+w=0$, $24^{\prime}$

(g) $\quad l x+\frac{y}{m}+n z+w=0$, .14 .23 .56

(万) $\quad l x+m y+\frac{z}{n}+w=0$, $43^{\prime}$

(x) $\quad x+\frac{l(p-\alpha)+2 m n}{p+\beta} w=0$, 12. 35.46

(y) $\quad y+\frac{m(p-\alpha)+2 n l}{p+\beta} w=0$, $52^{\prime}$

(z) $\quad z+\frac{n(p-\alpha)+2 l m}{p+\beta} w=0$, $15^{\prime}$

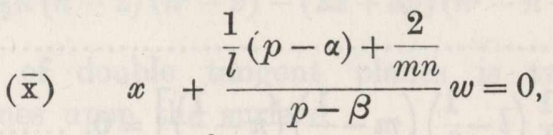
12.36 .45

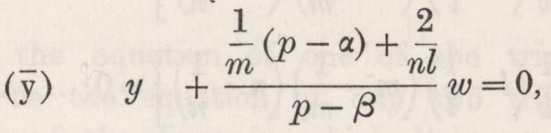
$62^{\prime}$

$(\bar{z}) \quad z+\frac{\frac{1}{n}(p-\alpha)+\frac{2}{l m}}{p-\beta} w=0$, $16^{\prime}$

(1) $-\frac{2 n}{m(p-\alpha)} x+\frac{1}{m} y+n z+w=0$, $56^{\prime}$

(m) $\quad l x-\frac{2 l}{n(p-\alpha)} y+\frac{1}{n} z+w=0$ $45^{\prime}$

(n) $\quad \frac{1}{l} x+m y-\frac{2 m}{l(p-\alpha)} z+w=0$, $4{ }^{\prime} 6$

(1) $\quad-\frac{2 m}{n(p-\alpha)} x+m y+\frac{1}{n} z+w=0$, 15. 26.34

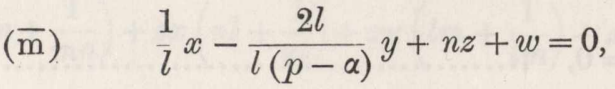
16.24 .35

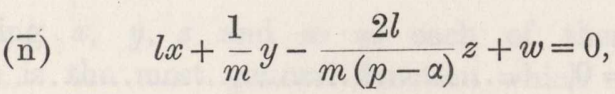
14.25 .36

(1) $-\frac{n(p-\alpha)}{2 m} x+\frac{y}{m}+n z+w=0$,

(m, $\quad l x-\frac{l(p-\alpha)}{2 n} y+\frac{1}{n} z+w=0$, $46^{\prime}$

(n) $\quad \frac{1}{l} x+m y-\frac{m(p-\alpha)}{2 l} z+w=0$, $4^{\prime} 5$ 
(1) $) \quad-\frac{m(p-\alpha)}{2 n} x+m y+\frac{1}{n} z+w=0$,

$\left(\overline{\mathrm{m}}_{l}\right) \quad \frac{1}{l} x-\frac{n(p-\alpha)}{2 l} y+n z+w=0$,

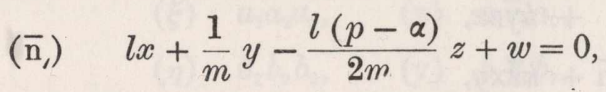

(p) $-\frac{2 x}{p-\alpha}+n y+m z+\left[m n(p-\alpha)-2 l\left(1-m^{2}-n^{2}\right)\right] \frac{w}{p+\beta}=0$,

(q) $\quad n x-\frac{2 y}{p-\alpha}+l z+\left[n l(p-\alpha)-2 m\left(1-n^{2}-l^{2}\right)\right] \frac{w}{p+\beta}=0$,

(r) $\quad m x+l y-\frac{2 z}{p-\alpha}+\left[\operatorname{lm}(p-\alpha)-2 n\left(1-l^{2}-m^{2}\right)\right] \frac{w}{p+\beta}=0, \ldots$

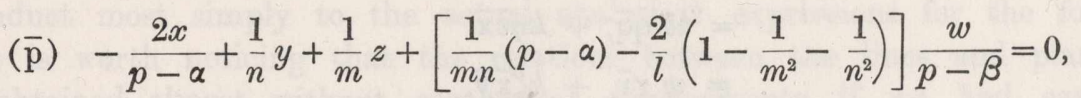
$26^{\prime}$

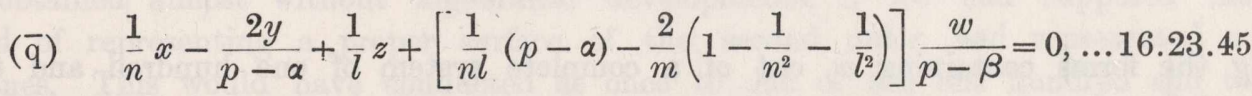

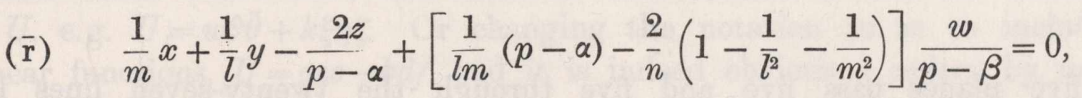

(p, $-\frac{p-\alpha}{2} x+\frac{y}{n}+\frac{z}{m}-\operatorname{lmn}\left[\frac{1}{l}\left(1-\frac{1}{m^{2}}-\frac{1}{n^{2}}\right)(p-\alpha)-\frac{2}{m n}\right] \frac{w}{p+\beta}=0$,

(q) $\frac{x}{n}-\frac{p-\alpha}{2} y+\frac{z}{l}-\operatorname{lm} n\left[\frac{1}{m}\left(1-\frac{1}{n^{2}}-\frac{1}{l^{2}}\right)(p-\alpha)-\frac{2}{n l}\right] \frac{w}{p+\beta}=0, \ldots 15.23 .46$

(r) $\quad \frac{x}{m}+\frac{y}{l}-\frac{p-\alpha}{2} z-\operatorname{lmn}\left[\frac{1}{n}\left(1-\frac{1}{l^{2}}-\frac{1}{m^{2}}\right)(p-\alpha)-\frac{2}{l m}\right] \frac{w}{p+\beta}=0$,

$(\bar{p})-\frac{p-\alpha}{2} x+n y+m z-\frac{1}{l m n}\left[l\left(1-m^{2}-n^{2}\right)(p-\alpha)-2 m n\right] \frac{w}{p-\beta}=0$,

(市) $\quad n x-\frac{p-\alpha}{2} y+l z-\frac{1}{l m n}\left[m\left(1-n^{2}-l^{2}\right)(p-\alpha)-2 n l\right] \frac{w}{p-\beta}=0$,

$$
m x+l y-\frac{p-\alpha}{2} z-\frac{1}{l m n}\left[n\left(1-l^{2}-m^{2}\right)(p-\alpha)-2 l m\right] \frac{w}{p-\beta}=0 \ldots 13.26 .45
$$

In fact, representing the several functions on the left-hand side of these equations respectively by the letters placed opposite to them respectively, the function $U$ is expressible in the sixteen forms following

$$
\begin{aligned}
U & =w \mathrm{f} \overline{\mathrm{f}}+k \xi y z \\
& =w \bar{g} \bar{g}+k \eta z x \\
& =w \mathrm{~h} \bar{h}+k \zeta x y \\
& =w \theta \bar{\theta}+k \xi \eta \zeta
\end{aligned}
$$

c. 


$$
\begin{aligned}
& =w \mathrm{l} \overline{\mathrm{l}},+k \overline{\mathrm{y}} x, \\
& =w \mathrm{~m} \overline{\mathrm{m}}, k \mathrm{z} \overline{\mathrm{x}} y, \\
& =w \mathrm{n} \overline{\mathrm{n}},+k \mathrm{x} \overline{\mathrm{y}} z, \\
& =w \mathrm{l}, \mathrm{l}+k \overline{\mathrm{y} z} x, \\
& =w \mathrm{~m}, \overline{\mathrm{m}}+k \overline{\mathrm{z} x} y, \\
& =w \mathrm{n}, \overline{\mathrm{n}}+k \overline{\mathrm{x}} \mathrm{y} z \\
& =w \mathrm{pp},+k \xi \mathrm{yz}, \\
& =w \mathrm{qq}+k \eta \mathrm{zx}, \\
& =w \mathrm{rr},+k \zeta \mathrm{xy}, \\
& =w \mathrm{p} \overline{\mathrm{p}},+k \xi \overline{\mathrm{y}} \overline{\mathrm{z}}, \\
& =w \mathrm{q} \overline{\mathrm{q}},+k \eta \overline{\mathrm{z}} \overline{\mathrm{x}}, \\
& =w \mathrm{r} \overline{\mathrm{r}},+k \overline{\mathrm{x}} \overline{\mathrm{y}},
\end{aligned}
$$

(being the forms containing $w$, out of a complete system of one hundred and twenty different forms).

The forty-five planes pass five and five through the twenty-seven lines in the following manner:

$\left(a_{1}\right)(w, x, \xi, \mathrm{x}, \overline{\mathrm{x}}) \ldots 12, \quad\left(a_{4}\right)\left(x, \mathrm{~g}, \overline{\mathrm{h}}, \mathrm{l}, \overline{\mathrm{I}}_{1}\right) \ldots 34, \quad\left(a_{7}\right)(\mathrm{x}, \mathrm{m}, \mathrm{n}, \mathrm{q}, \mathrm{r}) \ldots 46$, $\left(b_{1}\right)(w, y, \eta, \mathrm{y}, \overline{\mathrm{y}}) \ldots \mathbf{2}^{\prime}, \quad\left(b_{4}\right)\left(y, \mathrm{~h}, \overline{\mathrm{f}}, \overline{\mathrm{m}}, \overline{\mathrm{m}}_{\imath}\right) \ldots 2 \mathbf{2 4}, \quad\left(b_{7}\right)\left(\mathrm{y}, \mathrm{n}_{1}, \mathrm{l}, \mathbf{r}, \mathrm{p}\right) \ldots \quad 5$, $\left(c_{1}\right)(w, z, \zeta, \mathrm{z}, \overline{\mathrm{z}}) \ldots 1, \quad\left(c_{4}\right)\left(z, \mathrm{f}, \overline{\mathrm{g}}, \overline{\mathrm{n}}, \overline{\mathrm{n}}_{1}\right) \ldots 14, \quad\left(c_{7}\right)(\mathrm{z}, \mathrm{l}, \mathrm{m}, \mathrm{p}, \mathrm{q}) \ldots 5^{\prime}$, $\left(a_{2}\right)(\xi, \overline{\mathrm{f}}, \theta, \overline{\mathrm{p}}, \mathrm{p},) \ldots 2, \quad\left(a_{5}\right)(x, \overline{\mathrm{g}}, \mathrm{h}, \mathrm{l}, \mathrm{l},) \ldots 56, \quad\left(a_{8}\right)\left(\overline{\mathrm{x}}, \overline{\mathrm{m}}_{l}, \overline{\mathrm{n}}, \overline{\mathrm{q}}_{1}, \overline{\mathrm{r}}\right) \ldots 36$, $\left(b_{2}\right)\left(\eta, \overline{\mathrm{g}}, \theta, \overline{\mathrm{q}}, \mathrm{q}_{1}\right) \ldots 23, \quad\left(b_{5}\right)\left(y, \overline{\mathrm{h}}, \mathrm{f}, \mathrm{m}, \mathrm{m}_{\iota}\right) \ldots 4, \quad\left(b_{8}\right)\left(\overline{\mathrm{y}}, \overline{\mathrm{n}}_{1}, \overline{\mathrm{l}}, \overline{\mathrm{r}}_{1}, \overline{\mathrm{p}}\right) \ldots 26$, $\left(c_{2}\right)(\zeta, \overline{\mathrm{h}}, \theta, \overline{\mathrm{r}}, \mathrm{r},) \ldots 3^{\prime}, \quad\left(c_{5}\right)(z, \overline{\mathrm{f}}, \mathrm{g}, \mathrm{n}, \mathrm{n},) \ldots 4^{\prime}, \quad\left(c_{8}\right)(\overline{\mathrm{z}}, \overline{\mathrm{I}}, \overline{\mathrm{m}}, \overline{\mathrm{p}}, \overline{\mathrm{q}}) \ldots 16$, $\left(a_{3}\right)\left(\xi, \mathrm{f}, \bar{\theta}, \mathrm{p}, \overline{\mathrm{p}}_{\imath}\right) \ldots \mathrm{1}^{\prime}, \quad\left(a_{6}\right)\left(\mathrm{x}, \mathrm{m}, \mathrm{n}_{\iota}, \mathrm{q}, \mathrm{r}_{1}\right) \ldots 35, \quad\left(a_{9}\right)\left(\overline{\mathrm{x}}, \mathrm{m}, \mathrm{n}_{\iota}, \overline{\mathrm{q}}, \overline{\mathrm{r}}_{\imath}\right) \ldots 45$, $\left(b_{3}\right)\left(\eta, \mathrm{g}, \bar{\theta}, \mathrm{q}, \overline{\mathrm{q}}_{1}\right) \ldots 3, \quad\left(b_{6}\right)(\mathrm{y}, \mathrm{n}, \mathrm{l}, \mathrm{r}, \mathrm{p},) \ldots 25, \quad\left(b_{9}\right)\left(\overline{\mathrm{y}}, \mathrm{n}, \mathrm{l}, \overline{\mathrm{r}}, \overline{\mathrm{p}}_{\imath}\right) \ldots 6$, $\left(c_{3}\right)\left(\zeta, \mathrm{h}, \bar{\theta}, \mathrm{r}, \overline{\mathrm{r}}_{1}\right) \ldots 13, \quad\left(c_{6}\right)\left(\mathrm{z}, \mathrm{l}, \mathrm{m}, \mathrm{p}, \mathrm{q}, \ldots 15, \quad\left(c_{9}\right)(\overline{\mathrm{z}}, \mathrm{l}, \mathrm{m}, \overline{\mathrm{p}}, \overline{\mathrm{q}}) \ldots 6^{\prime}\right.$,

where each line may be represented by the letter placed oppositive to the system of planes passing through it. The twenty-seven lines lie three and three upon the forty-five planes in the following manner:
(w) $\quad a_{1} b_{1} c_{1}$
(f) $a_{3} b_{5} c_{4}$,
(l) $\quad a_{5} b_{7} c_{9}$,
(p) $a_{3} b_{7} c_{6}$,
( $\theta) \quad a_{2} b_{2} c_{2}$
(g) $b_{3} c_{5} a_{4}$,
(m) $b_{5} c_{7} a_{9}$,
(q) $b_{3} c_{7} a_{6}$,
( $\theta) \quad a_{3} b_{3} c_{3}$,
(h) $c_{3} a_{5} b_{4}$,
(n) $c_{5} a_{7} b_{9}$,
(r) $c_{3} a_{7} b_{6}$, 

(x) $a_{1} a_{4} a_{5}$,
(f) $a_{2} b_{4} c_{5}$,
(i) $\quad a_{4} b_{8} c_{6}$,

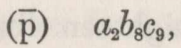
(y) $b_{1} b_{4} b_{5}$,
(g) $b_{2} c_{4} a_{5}$,
(im) $b_{4} c_{8} a_{6}$,

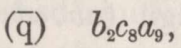
(z) $c_{1} c_{4} c_{5}$,
(द) $c_{2} a_{4} b_{5}$,

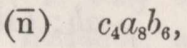

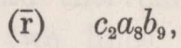

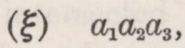
(x) $a_{1} a_{6} a_{7}$,
(1) $a_{5} b_{9} c_{7}$,
(p) $a_{2} b_{6} c_{7}$,
(च) $b_{1} b_{2} b_{3}$,
(y) $b_{1} b_{6} b_{7}$,
(m) $b_{5} c_{9} a_{7}$,
(q) $b_{2} c_{6} a_{7}$,

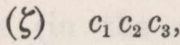
(z) $c_{1} c_{6} c_{7}$,
(n) $c_{5} a_{9} b_{7}$,
(r) $c_{2} a_{6} b_{7}$,

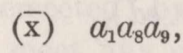

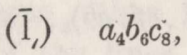
(市) $a_{3} b_{9} c_{8}$,
(y) $b_{1} b_{8} b_{9}$,

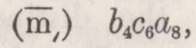

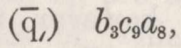
(z) $c_{1} c_{8} c_{9}$,
(n) $\quad c_{4} a_{6} b_{8}$,
(i, $c_{3} a_{9} b_{8}$.

The preceding method was the one that first occurred to me, and which appears to conduct most simply to the actual analytical expressions for the forty-five planes; but it is worth noticing that the relations between the lines and planes might have been obtained almost without algebraical developments, if we had supposed that $P$, instead of representing a proper surface of the second order, had represented a pair of planes. This would have conducted at once to one of the one hundred and twenty forms $U$, e.g. $U=w \theta \bar{\theta}+k \xi \eta \zeta$. Or changing the notation so as to include $k$ in one of the linear functions, $U=a c e-b d f$, and it is indeed obvious $a$ priori, by merely reckoning the number of arbitrary constants, that any function of the third order can be put under this form. If we suppose $a=\mu b$ to be the equation of one of the triple tangent planes through the intersection of the planes $a$ and $b$, the plane $a=\mu b$ meets the surface in the same lines in which it meets the hyperboloid $\mu c e-d f=0$, that is, the two lines in the plane are generating lines of different species, and consequently one of them meets the pair of lines $c d$ and $e f$, and the other of them meets the pair of lines $c f$ and $d e$ (where $c d$ represents the line of intersection of the planes $c=0, d=0$, \&c.). This suggests a notation for the lines in question, viz. each line may be represented by the three lines which it meets, or by the symbols ab.cd.ef and ab.cf.de. Or observing that $\mu$ has three values, and that the same considerations apply mutatis mutandis to the planes through $b c$ and $c a$, the whole system of lines may be represented by the notation,

$\begin{array}{ccc}a b, & a d, & a f, \\ c b, & c d, & c f, \\ e b, & e d, & e f, \\ (a b \cdot c d \cdot e f)_{1}, & (a b \cdot c d \cdot e f)_{2}, & (a b \cdot c d \cdot e f)_{3}, \\ (a d \cdot c f \cdot e b)_{1}, & (a d \cdot c f \cdot e b)_{2}, & (a d \cdot c f \cdot e b)_{3}, \\ (a f \cdot c b \cdot e d)_{1}, & (a f \cdot c b \cdot e d)_{2}, & (a f \cdot c b \cdot e d)_{3}, \\ (a b \cdot c f \cdot e d)_{1}, & (a b \cdot c f \cdot e d)_{2}, & (a b \cdot c f \cdot e d)_{3}, \\ (a d \cdot c b \cdot e f)_{1}, & (a d \cdot c b \cdot e f)_{2}, & (a d \cdot c b \cdot e f)_{3}, \\ (a f \cdot c d \cdot e b)_{1}, & (a f \cdot c d \cdot e b)_{2}, & (a f \cdot c d \cdot e b)_{3},\end{array}$


where the last eighteen lines have been divided into two systems of nine each. The five planes through $(a b . c d . e f)_{1}$ may be considered as cutting the surface in

$$
\begin{aligned}
a b ;(a b . c f . e d)_{1}, \\
c d ;(a f . c d . e b)_{1}, \\
e f ;(a d . c b . e f)_{1}, \\
(a d . c f . e b)_{2} ;(a f . c b . e d)_{3}, \\
(a d . c f . e b)_{3} ;(a f . c b . e d)_{2},
\end{aligned}
$$

(which supposes however that the distinguishing suffixes 1, 2, 3, are added to the different planes according to a certain rule). And similarly for the lines in the planes through the other lines represented by symbols of the like form. The five planes through $a b$ intersect the surface in the lines

$$
\begin{array}{cc}
c b, & e b, \\
a d, & a f, \\
(a b . c d . e f)_{1}, & (a b \cdot c f \cdot e d)_{1}, \\
(a b . c d \cdot e f)_{2}, & (a b \cdot c f \cdot e d)_{2}, \\
(a b . c d \cdot e f)_{3}, & (a b . c f . e d)_{3},
\end{array}
$$

and similarly for the planes through the other lines represented by symbols of a like form.

Observing that $\xi, \eta, \zeta$, correspond to $b, d, f$, and $w, \theta, \bar{\theta}$, to $a, c, e$ respectively, $a b$ corresponds to the intersection of $w$ and $\xi$, i.e. to $a_{1}$, \&c.; also (ab.cd.ef) $)_{1}$, $(a b . c d . e f)_{2},(a b . c d . e f)_{3}$ correspond to three lines meeting $a_{1}, b_{2}$, and $c_{3}$, i.e. to $a_{5}, a_{7}, a_{9}$, \&c. ; and the system of the twenty-seven lines as last written down corresponds to the system,

$$
\begin{array}{lll}
a_{1}, & b_{1}, & c_{1}, \\
a_{2}, & b_{2}, & c_{2}, \\
a_{3}, & b_{3}, & c_{3}, \\
a_{5}, & a_{7}, & a_{9}, \\
b_{5}, & b_{7}, & b_{9}, \\
c_{5}, & c_{7}, & c_{9}, \\
a_{4}, & a_{6}, & a_{8}, \\
b_{4}, & b_{6}, & b_{8}, \\
c_{4}, & c_{6}, & c_{8} .
\end{array}
$$

The investigations last given are almost complete in themselves as the geometrical theory of the subject: there is however some difficulty in seeing $\grave{a}$ priori the nature of the correspondence between the planes which determines which are the planes which ought to be distinguished with the same one of the symbolic numbers, $1,2,3$. 
There is great difficulty in conceiving the complete figure formed by the twentyseven lines, indeed this can hardly I think be accomplished until a more perfect notation is discovered. In the mean time it is easy to find theorems which partially exhibit the properties of the system. For instance, any two lines, $a_{1}, b_{2}$, which do not meet are intersected by five other lines, $a_{2}, b_{1}, a_{5}, a_{7}, a_{9}$, (no two of which meet). Any four of these last-mentioned lines are intersected by the lines $a_{1}, b_{2}$ and no other lines, but any three of them, e.g. $a_{5}, a_{7}, a_{9}$, are intersected by the lines $a_{1}, b_{2}$, and by some third line (in the case in question the line $c_{3}$ ). Or generally any three lines, no two of which meet, are intersected by three other lines, no two of which meet. Again, the lines which do not meet any one of the lines $a_{5}, a_{7}, a_{9}$, are $a_{2}, a_{3}, b_{3}, b_{1}, c_{1}, c_{2}$ : these lines form a hexagon, the pairs of the opposite sides of which, $a_{2}, b_{1} ; a_{3}, c_{1}$; $b_{3}, c_{2}$, are met by the pairs $a_{1}, b_{2} ; c_{3}, a_{1}$ and $a_{1}, b_{2}$, respectively, viz. by pairs out of the system of three lines intersecting the system $a_{5}, a_{7}, a_{9}$. And the lines $a_{5}, a_{7}, a_{9}$ may be considered as representing any three lines no two of which meet. Again, consider three lines in the same triple tangent plane, e.g. $a_{1}, b_{1}, c_{1}$, and the hexahedron formed by any six triple tangent planes passing two and two through these lines, e.g. the planes $x, y, z, \xi, \eta, \zeta$. These planes contain (independently of the lines $a_{1}, b_{1}, c_{1}$ ) the twelve lines $a_{2}, a_{3}, a_{4}, a_{5}, b_{2}, b_{3}, b_{4}, b_{5}, c_{2}, c_{3}, c_{4}, c_{5}$. Consider three contiguous faces of the hexahedron, e.g. $x, y, z$, the lines in these planes, viz. $a_{4}, b_{5}, c_{4}, a_{5}, b_{4}, c_{5}$, form a hexagon the opposite sides of which intersect in a point, or in other words these six lines are generating lines of a hyperboloid. The same property holds for the systems $x, \eta, \zeta ; \xi, y, \zeta ; \xi, \eta, z$. But for the system $\xi, \eta, \zeta$, the six lines are $a_{2}, b_{2}, c_{2}$, and $a_{3}, b_{3}, c_{3}$, which form two triangles, and similarly for the systems $\xi, y, z ; x, \eta, z ;$ and $x, y, \zeta$; so that the twelve lines form four hexagons (the opposite sides of which intersect) circumscribed round four of the angles of the hexahedron, and four pairs of triangles about the opposite four angles of the hexahedron. The number of such theorems might be multiplied indefinitely, and the number of different combinations of lines or planes to which each theorem applies is also very considerable.

Consider the four planes $x, \xi, \mathrm{x}, \overline{\mathrm{x}}$, and represent for a moment the equations of these planes by $x+A w=0, x+B w=0, x+C w=0, x+D w=0$, so that

$$
A=0, \quad B=\frac{1}{k}\left(m-\frac{1}{m}\right)\left(n-\frac{1}{n}\right), \quad C=\frac{l(p-\alpha)+2 m n}{p+\beta}, \quad D=\frac{\frac{1}{l}(p-\alpha)+\frac{2}{m n}}{p-\beta} .
$$

By the assistance of

$$
B-C=\frac{-1}{\operatorname{lmn}\left(p^{2}-\beta^{2}\right)}[\ln (p-\alpha)+2 m][\operatorname{lm}(p-\alpha)+2 n]
$$

it is easy to obtain

$$
\frac{(A-C)(D-B)}{(A-D)(B-C)}=\operatorname{lmn} \frac{p-\beta[l(p-\alpha)+2 m n][m(p-\alpha)+2 n l][n(p-\alpha)+2 l m]}{p+\beta},
$$


which remains unaltered for cyclical permutations of $l, m, n$, i.e. the anharmonic ratio of $x, \xi, \mathrm{x}, \overline{\mathrm{x}}$ is the same as that of $y, \eta, \mathrm{y}, \overline{\mathrm{y}}$, or $z, \zeta, \mathrm{z}, \overline{\mathrm{z}}$; there is of course no correspondence of $x$ to $y$ or $\xi$ to $\eta$, \&c., the correspondence is by the general properties of anharmonic ratios, a correspondence of the system $x, \xi, \mathrm{x}, \overline{\mathrm{x}}$, to any one of the systems $(y, \eta, \mathrm{y}, \overline{\mathrm{y}})$, or $(\eta, y, \overline{\mathrm{y}}, \mathrm{y})$, or $(\mathrm{y}, \overline{\mathrm{y}}, y, \eta)$, or $(\overline{\mathrm{y}}, \mathrm{y}, \eta, y)$, indifferently. The theorems may be stated generally as follows: "Considering two lines in the same triple tangent plane, the remaining triple tangent planes through these two lines respectively are homologous systems."

Suppose the surface of the third order intersected by an arbitrary plane. The curve of intersection is of course one of the third order, and the positions upon this curve of six of the points in which it is intersected may be arbitrarily assumed. Let these points be the points in which the plane is intersected by the lines $a_{1}, b_{1}, a_{6}, b_{6}, c_{6}, a_{8}$; or as we may term them, the points $a_{1}, b_{1}, a_{6}, b_{6}, c_{6}, a_{8} .\left(^{1}\right)$ The point $c_{1}$ is of course the point in which the line $a_{1} b_{1}$ intersects the curve. The straight lines $a_{4} b_{6} c_{8}, b_{4} c_{6} a_{8}, c_{4} a_{6} b_{8}$, and $a_{4} b_{8} c_{6}, b_{4} c_{8} a_{6}, c_{4} a_{8} b_{6}$, show that $c_{4}$ and $b_{4}$ are the points in which $a_{8} b_{6}$, and $a_{8} c_{6}$ intersect the curve, and then $b_{8}$ and $c_{8}$ are determined as the intersections of $a_{6} c_{4}, a_{6} b_{4}$ with the curve. The intersection of the lines $b_{6} c_{8}$ and $b_{8} c_{6}$ (which is known to be a point upon the curve by the theorem, every curve of the third order passing through eight of the points of intersection of two curves of the third order passes through the ninth point of intersection) is the point $a_{4}$. The systems $a_{4}, b_{4}, c_{4} ; a_{6}, b_{6}, c_{6} ; a_{8}, b_{8}, c_{8}$, determine the conjugate system $a_{5}, b_{5}, c_{5} ; a_{7}, b_{7}, c_{7} ; a_{9}, b_{9}, c_{9}$; by reason of the straight lines $a_{1} a_{4} a_{5}, b_{1} b_{4} b_{5}, c_{1} c_{4} c_{5} ; a_{1} a_{6} a_{7}, b_{1} b_{6} b_{7}, c_{1} c_{6} c_{7} ; a_{1} a_{8} a_{9}, b_{1} b_{8} b_{9}, c_{1} c_{8} c_{9}, v i z . a_{5}$ is the point where $a_{1} a_{4}$ intersects the curve, \&c. The relations of the systems $\left(a_{4}, b_{4}, c_{4} ; a_{5}, b_{5}, c_{5}\right),\left(a_{6}, b_{6}, c_{6}\right.$; $\left.a_{7}, b_{7}, c_{7}\right),\left(a_{8}, b_{8}, c_{8} ; a_{9}, b_{9}, c_{9}\right)$ to the system $a_{1}, b_{1}, c_{1} ; a_{2}, b_{2}, c_{2} ; a_{3}, b_{3}, c_{3}$ are precisely identical. It is only necessary to show how the points $a_{2}, b_{2}, c_{2} ; a_{3}, b_{3}, c_{3}$ of the latter system are determined by means of one of the former systems, suppose the system $a_{6}, b_{6}, c_{6} ; a_{7}, b_{7}, c_{7}$; and to discover a compendious statement of the relation between the two systems. The points $a_{1}, b_{1}, c_{1} ; a_{2}, b_{2}, c_{2} ; a_{3}, b_{3}, c_{3} ; a_{6}, b_{6}, c_{6} ; a_{7}, b_{7}, c_{7}$, are a system of fifteen points lying on the fifteen straight lines $a_{1} b_{1} c_{1}, a_{2} b_{2} c_{2}, a_{3} b_{3} c_{3}$, $a_{1} a_{2} a_{3}, b_{1} b_{2} b_{3}, c_{1} c_{2} c_{3}, a_{1} a_{6} a_{7}, b_{1} b_{6} b_{7}, c_{1} c_{6} c_{7}, a_{3} b_{7} c_{6}, b_{3} c_{7} a_{6}, c_{3} a_{7} b_{6}, a_{2} b_{6} c_{7}, b_{2} c_{6} a_{7}, c_{2} a_{6} b_{7}$, viz. the nine points $a_{1}, b_{1}, c_{1} ; a_{2}, b_{2}, c_{2} ; a_{3}, b_{3}, c_{3}$ are the points of intersection of the three lines $a_{1} b_{1} c_{1}, a_{2} b_{2} c_{2}, a_{3} b_{3} c_{3}$ with the three lines $a_{1} a_{2} a_{3}, b_{1} b_{2} b_{3}, c_{1} c_{2} c_{3}$, and the remaining six points form a hexagon $a_{6} b_{7} c_{6} a_{7} b_{6} c_{7}$, of which the diagonals $a_{6} a_{7}, b_{6} b_{7}, c_{6} c_{7}$ pass through the points $a_{1}, b_{1}, c_{1}$, respectively, the alternate sides $a_{6} b_{7}, c_{6} a_{7}$, and $b_{6} c_{7}$ pass through the points $c_{2}, b_{2}, a_{2}$ respectively, and the remaining alternate sides $b_{7} c_{6}, a_{7} b_{6}$, and $c_{7} a_{6}$ pass through the three points $a_{3}, b_{3}, c_{3}$ respectively. The fifteen points of such a system do not necessarily lie upon a curve of the third order, as will presently be seen: in the actual case however where all the points lie upon a given curve of the third order, and the points $a_{1}, b_{1}, c_{1} ; a_{6}, b_{6}, c_{6} ; a_{7}, b_{7}, c_{7}$ are known, $a_{2}, b_{2}, c_{2} ; a_{3}, b_{3}, c_{3}$ are the intersections of the curve with $b_{6} c_{7}, c_{6} a_{7}, a_{6} b_{7}, b_{7} c_{6}, c_{7} a_{6}, a_{7} b_{6}$ respectively, and the fact

1 In general, the point in which any line upon the surface intersects the plane in question may be represented by the symbol of the line, and the line in which any triple tangent plane intersects the plane in question may be represented by the symbol of the triple tangent plane: thus, $a_{1}, b_{1}, c_{1}$ are points in the line $a_{1} b_{1} c_{1}$, or in the line $w, \& c$. 
of the existence of the lines $a_{1} b_{1} c_{1}, a_{2} b_{2} c_{2}, a_{3} b_{3} c_{3}, a_{1} a_{2} a_{3}, b_{1} b_{2} b_{3}, c_{1} c_{2} c_{3}$ is an immediate consequence of the theorem quoted above with respect to curves of the third ordera theorem from which the entire system of relations between the twenty-seven points on the curve might have been deduced $a$ priori. But returning to the system of fifteen points, suppose the lines $a_{1} b_{1} c_{1}, a_{2} b_{2} c_{2}, a_{3} b_{3} c_{3}$, and $a_{1} a_{2} a_{3}, b_{1} b_{2} b_{3}$, and also the point $a_{6}$ to be given arbitrarily. The point $a_{7}$ lies on the line $a_{1} a_{6}$, suppose its position upon this line to be arbitrarily assumed (in which case, since the ten points $a_{1}, a_{2}, a_{3}$, $b_{1}, b_{2}, b_{3}, c_{1}, c_{2}, c_{3}$, are sufficient to determine a curve of the third order, there is no curve of the third order through these points and the point $a_{7}$ ). If the points

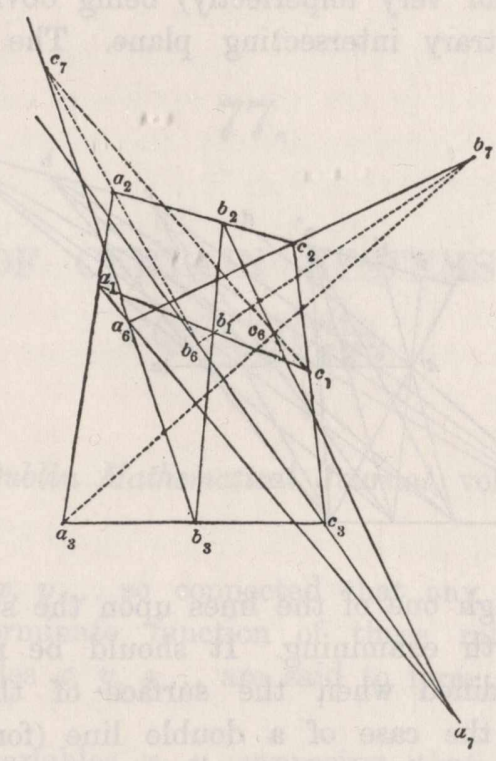

$b_{6}, c_{6}, b_{7}, c_{7}$ can be so determined that the sides of the quadrilateral $b_{6} b_{7} c_{6} c_{7}$, viz. $b_{6} b_{7}, b_{7} c_{6}, c_{6} c_{7}, c_{7} b_{6}$ pass through the points $b_{1}, a_{3}, c_{1}, a_{2}$ respectively, while the angles $b_{6}, b_{7}, c_{6}, c_{7}$ lie upon the lines $a_{7} c_{3}, a_{6} c_{2}, a_{7} b_{2}$ and $a_{6} b_{3}$ respectively, the required conditions will be satisfied by the fifteen points in question; and the solution of this problem is known. I have not ascertained whether in the case of an arbitrary position as above of the point $a_{7}$, it is possible to determine a complete system of twentyseven points lying three and three upon forty-five lines in the same manner as the twenty-seven points upon the curve of the third order; but it appears probable that this is the case, and to determine whether it be so or not, presents itself as an interesting problem for investigation.

Suppose that the intersecting plane coincides with one of the triple tangent planes. Here we have a system of twenty-four points, lying eight and eight in three lines; the twenty-four points lie also three and three in thirty-two lines, which last-mentioned lines therefore pass four and four through the twenty-four points. If we represent by $a, b, c, d, a^{\prime}, b^{\prime}, c^{\prime}, d^{\prime}$ and $a, b, c, d, a^{\prime}, b^{\prime}, c^{\prime}, d^{\prime}$, the eight points, and eight points 
which lie upon two of the three lines (the order being determinate), the systems of four lines which intersect in the eight points of the third line are

$$
\begin{aligned}
& (a \mathrm{a}, \quad b \mathrm{~b}, \quad c \mathrm{c}, \quad d \mathrm{~d}), \quad\left(a^{\prime} \mathrm{a}^{\prime}, \quad b^{\prime} \mathrm{b}^{\prime}, \quad c^{\prime} \mathrm{c}^{\prime}, \quad d^{\prime} \mathrm{d}^{\prime}\right) \text {; } \\
& \left(a \mathrm{~b}^{\prime}, \quad b \mathrm{a}^{\prime}, \quad c^{\prime} \mathrm{d}, \quad d^{\prime} \mathrm{c}\right), \quad\left(a^{\prime} \mathrm{b}, \quad b^{\prime} \mathrm{a}, \quad c \mathrm{~d}^{\prime}, \quad d \mathrm{c}^{\prime}\right) \text {; } \\
& \left(a c^{\prime}, \quad c a^{\prime}, \quad d^{\prime} \mathrm{b}, \quad b^{\prime} \mathrm{d}\right), \quad\left(a^{\prime} \mathrm{c}, \quad c^{\prime} \mathrm{a}, \quad b \mathrm{~d}^{\prime}, \quad d \mathrm{~b}^{\prime}\right) \text {; } \\
& \left(a \mathrm{~d}^{\prime}, \quad d \mathrm{a}^{\prime}, \quad b^{\prime} \mathrm{c}, \quad c^{\prime} \mathrm{b}\right), \quad\left(a^{\prime} \mathrm{d}, \quad d^{\prime} \mathrm{a}, \quad b \mathrm{c}^{\prime}, \quad c \mathrm{~b}^{\prime}\right) \text { : }
\end{aligned}
$$

the principle of symmetry made use of in this notation (which however represents the actual symmetry of the system very imperfectly) being obviously entirely different from that of the case of an arbitrary intersecting plane. The transition case where the

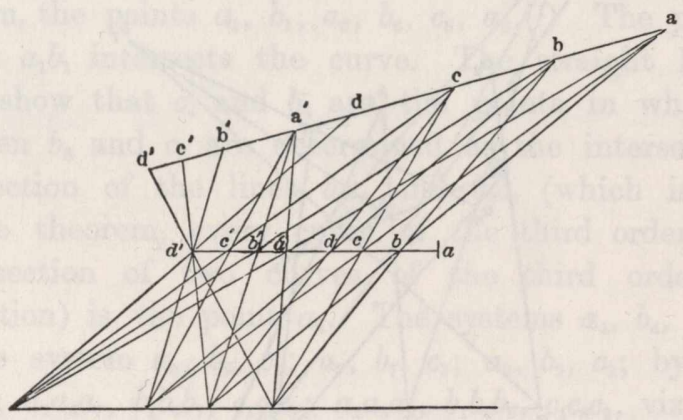

intersecting plane passes through one of the lines upon the surface (and is thus a double tangent plane) would be worth examining. It should be remarked that the preceding theory is very materially modified when the surface of the third order has one or more conical points; and in the case of a double line (for which the surface becomes a ruled surface) the theory entirely ceases to be applicable. I may mention in conclusion that the whole subject of this memoir was developed in a correspondence with $\mathrm{Mr}$ Salmon, and in particular, that I am indebted to him for the determination of the number of lines upon the surface and for the investigations connected with the representation of the twenty-seven lines by means of the letters $a, c, e, b, d, f$, as developed above. 